Article

\title{
Impact of Temporary Nitrogen Deprivation on Tomato Leaf Phenolics
}

\section{Camille Bénard ${ }^{1}$, Frédéric Bourgaud ${ }^{2}$ and Hélène Gautier ${ }^{1}{ }^{*}$}

1 INRA UR 1115 Horticultural Plants and Culture Systems (PSH), Domaine Saint Paul, F-84914 Avignon, France; E-Mail: camille.benard@avignon.inra.fr

2 UMR 1121 University of Lorraine (INPL)-INRA Agronomy and Environment Nancy-Colmar, ENSAIA 2, avenue de la forêt de Haye F-54505 Vandœuvre-lès-Nancy, France;

E-Mail: frederic.bourgaud@ensaia.inpl-nancy.fr

* Author to whom correspondence should be addressed; E-Mail: helene.gautier@avignon.inra.fr; Tel.: +334-32-72-23-45; Fax: +334-32-72-22-80.

Received: 9 September 2011; in revised form: 7 November 2011 / Accepted: 8 November 2011 Published: 16 November 2011

\begin{abstract}
Reducing the use of pesticides represents a major challenge of modern agriculture. Plants synthesize secondary metabolites such as polyphenols that participate in the resistance to parasites. The aim of this study was to test: (1) the impact of nitrogen deficiency on tomato (Solanum lycopersicum) leaf composition and more particularly on two phenolic molecules (chlorogenic acid and rutin) as well as on the general plant biomass; and (2) whether this effect continued after a return to normal nitrogen nutrition. Our results showed that plants deprived of nitrogen for 10 or 19 days contained higher levels of chlorogenic acid and rutin than control plants. In addition, this difference persisted when the plants were once again cultivated on a nitrogen-rich medium. These findings offer interesting perspectives on the use of a short period of deprivation to modulate the levels of compounds of interest in a plant.
\end{abstract}

Keywords: chlorogenic acid; rutin; nitrogen deprivation; phenolic compound; tomato

\section{Introduction}

During their development, plants produce different vital metabolites that are commonly referred to as primary metabolites. They also synthesize numerous compounds qualified as "secondary", the 
functions of which have not always been identified yet but which remain fundamental, notably regarding the adaptation of plants to their environment. Of these secondary compounds, polyphenols are, amongst others, involved in the mechanisms of resistance to biotic and abiotic stresses. In tomato, leaves are a site of infection by parasites, and the damage caused can have dramatic issues on plant productivity, reducing plant photosynthesis and inducing a loss of fruit yield. Chlorogenic acid and rutin are the principal phenolic compounds found in tomato leaves [1]. It has been shown that these compounds intervene directly in resistance to fungal and bacterial pathogens [2-4]. However, their protective role against herbivores remains controversial [5-7].

A reduction in the use of pesticides represents a major challenge for modern agriculture, particularly in the context of preserving the environment and human health. For this reason, improving the resistance of plants to parasite attacks by modulating their cultivation conditions offers interesting opportunities [8]. In particular, a deficit in mineral elements has often been employed to study the regulation and formation of secondary metabolites, including phenolic compounds $[9,10]$. Several studies showed that a reduction in nitrogen nutrition increases the levels of phenolic compounds in leaves [1,11-14]. Nitrogen deprivation also induces negative effects on plant growth [15,16] and yield [17]. It would therefore be interesting to determine cultivation methods that could improve plant resistance without affecting production; for example by subjecting plants to moderate stress or by alternating periods of environmental restriction with periods of optimum conditions. This latter strategy suggests knowing whether these modifications to secondary metabolism will persist once the environmental restrictions have been lifted, allowing the plant to increase its resistance to other types of biotic or abiotic stress.

Such responses had already been observed in the tomato and grasses when they were subjected to successive periods of water stress $[18,19]$. These studies showed that moderate water stress induced an acclimatization of plants that allowed them subsequently to better tolerate more severe water stress. On the other hand, following a period of nitrogen deficit, Scheible and co-workers [20] showed different evolution pattern of phenylpropanoïd metabolites in Arabidopsis plantlets replaced on a nitrogen-rich substrate. The behavior of tomato plants following a return to non-limiting conditions after an episode of nitrogen deprivation has not been studied. The aim of the present study was therefore to determine the strategy of this plant in the event of temporary nitrogen deprivation followed by a return to a nitrogen-rich substrate. Biomass growth, tissue contents in carbon and nitrogen and the accumulation of phenolic compounds were thus determined following a period of deprivation and five days after a return to normal conditions.

\section{Results}

\subsection{Effects on Plant Biomass}

The distribution of plant biomass was modified by the period of deprivation (Figure 1). The proportion of fresh matter allocated to the roots was significantly greater in plants growing under nitrogen deprivation (39\%) than in plants growing on the rich medium $(26 \%)$. This increase in the fresh matter allocated to the roots was achieved mainly to the detriment of the stems, as the fresh matter assigned to leaves was only slightly modified. Similar results were observed during experiment $\mathrm{B}$, when plants were subjected to a longer period of deprivation (results not presented). 
Figure 1. Percentage distribution of fresh biomass produced between the three plant compartments: roots, stem and leaves. Plants belong to the experiment A, and were harvested at the end of the period of $\mathrm{N}$ deprivation (Harvest 1, Figure 2). (a) Plants grown for 10 days on a rich medium $\left(7 \mathrm{mM} \mathrm{NO}_{3}{ }^{-}\right)$or (b) plants grown for 10 days on nitrogen deficient medium $\left(0 \mathrm{mM} \mathrm{NO}_{3}{ }^{-}\right)$. Stars $(*)$ indicate significant difference between the two treatments, i.e., plants grown on 7 or $0 \mathrm{mM}$; NS, not significant.
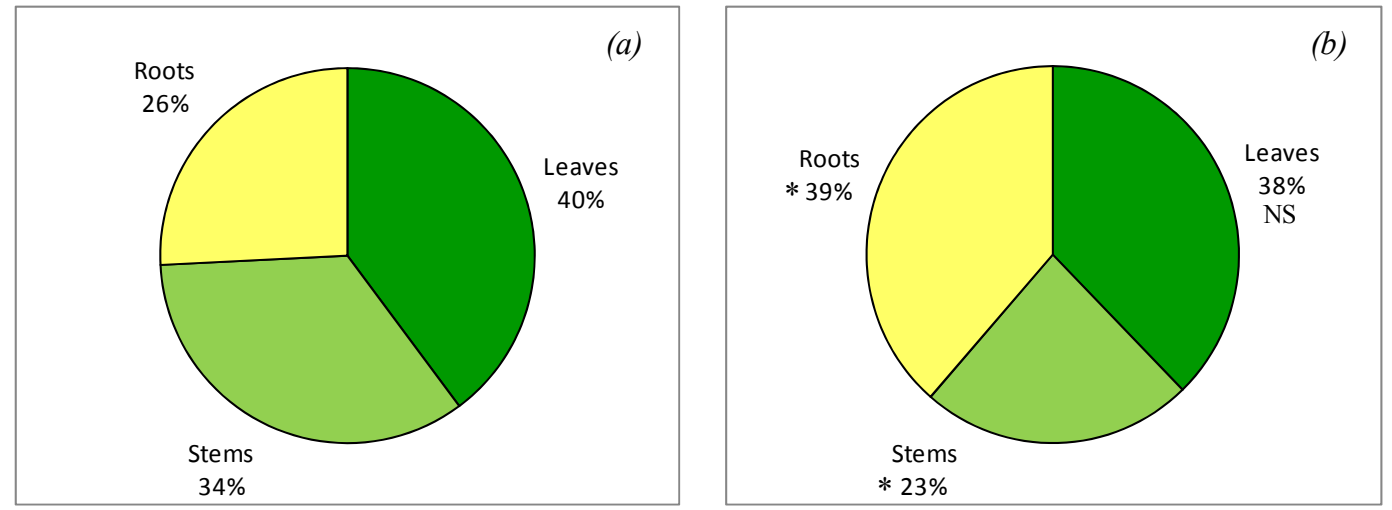

Figure 2. Simplified presentation of the experimental protocol used to study the effect of temporary nitrogen deprivation in tomato. During the two experiments, after 10 days of acclimatization at $7 \mathrm{mM} \mathrm{NO}_{3}{ }^{-}, 8$ plants were placed in a situation of deprivation (treated plants at $0 \mathrm{mM} \mathrm{NO}_{3}{ }^{-}$) and 8 control plants were placed on a rich medium containing $7 \mathrm{mM}$. 4 treated plants and 4 control plants were harvested following the period of deprivation (Harvest 1: H1). The four remaining plants per treatment were cultivated on the rich medium for a further 5 days before being harvested (Harvest 2: H2). During experiment A, the plants were deprived for 10 days, and during experiment $\mathrm{B}$, for 19 days.

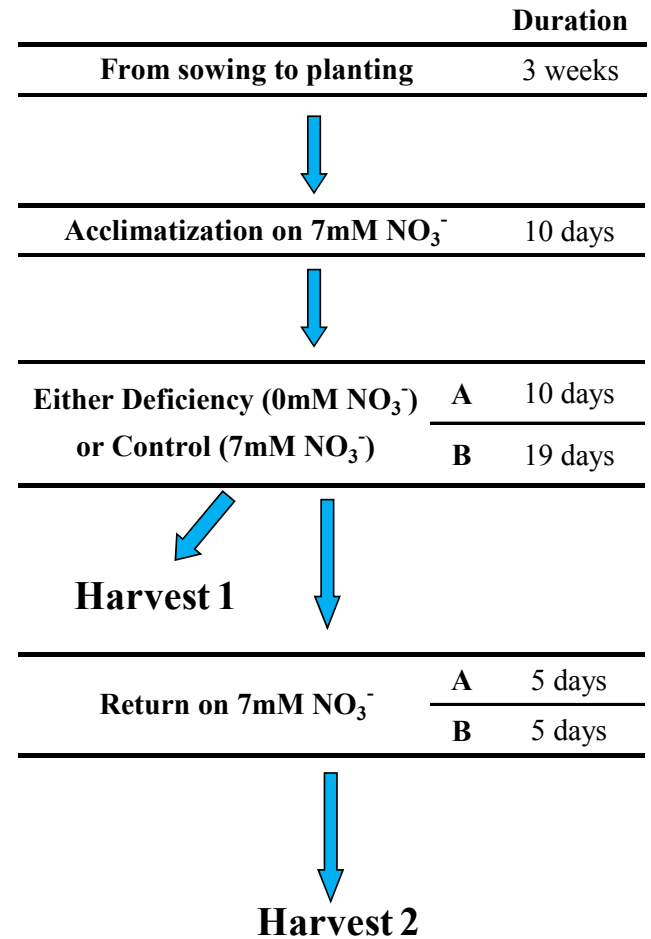


At the end of the experimental period, a reduction of approximately $50 \%$ in total biomass was observed in treated plants (Figure 3). After a return to the rich medium for five days, the growth of plants that had been nitrogen-deprived was not sufficient to make up for their delayed development. After five days of a return to $7 \mathrm{mM}$, the reduction in fresh matter in previously treated plants compared to controlled plants, ranged from $12 \%-31 \%$ in the roots, $59 \%-61 \%$ in the stems and $68 \%-73 \%$ in the leaves, the longer period of nitrogen deprivation during experiment $\mathrm{B}$ inducing a more marked reduction in biomass.

Figure 3. Evolution of fresh weight in leaves, stems, roots and total biomass during experiments A (Harvest 1 at 10 and Harvest 2 at 15 days) and B (H1 at 19 and $\mathrm{H} 2$ at 24 days). Data are mean \pm SE calculated on four plants, and expressed as g per plant. Stars indicate significant effect of nitrogen. ${ }^{*}, P<0.05$; **, $P<0.01$; ***, $P<0.001$; I, interaction between container and nitrogen effect.
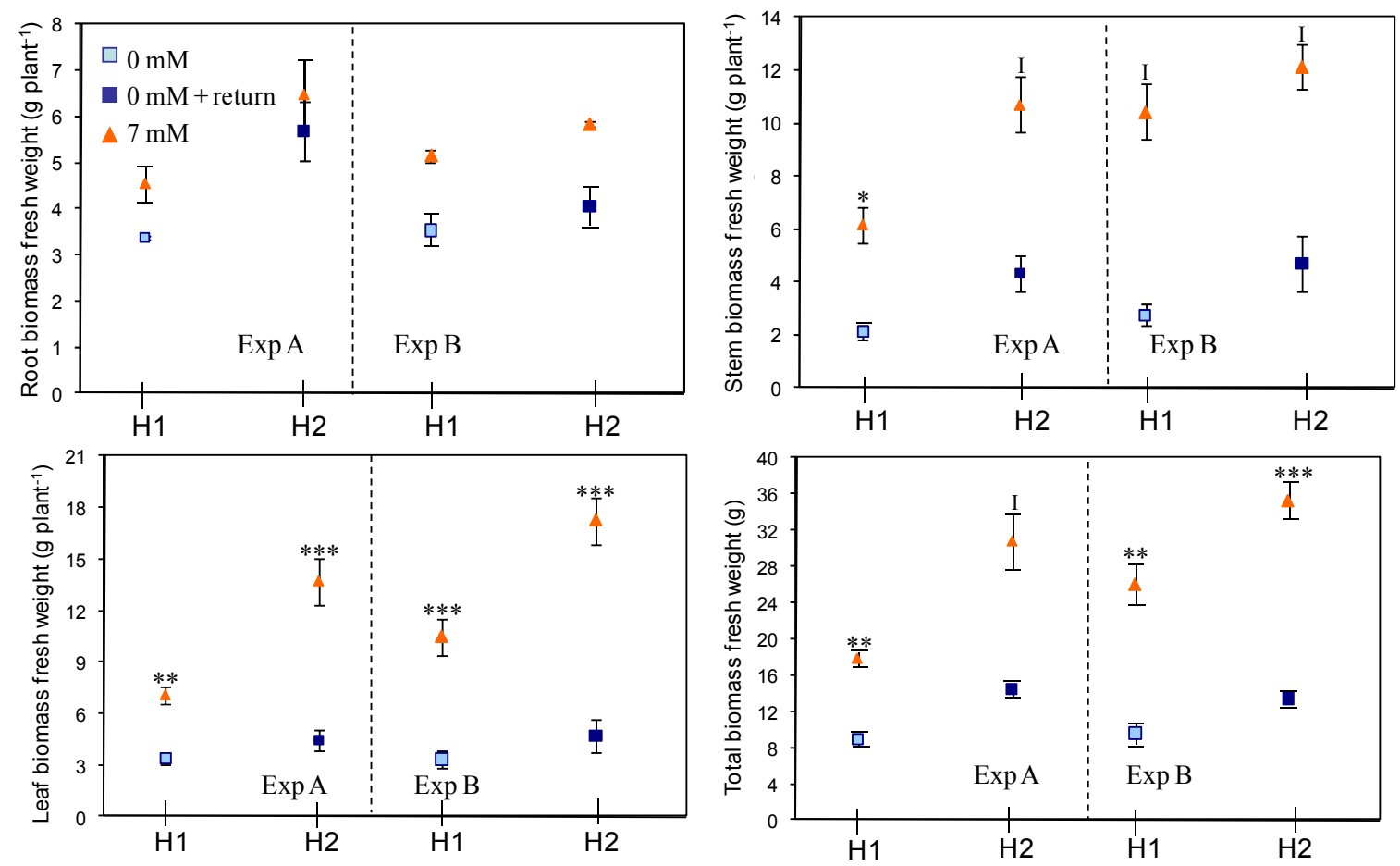

\subsection{Effects on Plant Composition}

Nitrogen deprivation induced a marked reduction in the percentage of total nitrogen in leaves that reached $62 \%$ and $71 \%$, respectively, during experiments $\mathrm{A}$ and $\mathrm{B}$ (Figure 4). After a return to a rich medium, the percentage variations were reduced to $21 \%$ and $18 \%$, respectively, thus demonstrating the very rapid nitrogen uptake dynamic of tissues. However, the periods of deprivation and the return to a rich medium did not affect tissue carbon contents. 
Figure 4. Evolution of contents in phenolic compounds chlorogenic acid and rutin in mg.g ${ }^{-1}$ dry weight and contents in total nitrogen and carbon in percentage of dry weight $(\%)$, during experiments A and B. Data are mean \pm SE calculated on four plants. Legends are similar to Figure 3. Stars indicate significant effect of nitrogen. NS, not significant, i.e., $P>0.1 ; *, P<0.05 ; * *, P<0.01 ; * * * P<0.001$; I, interaction between container and nitrogen effect.
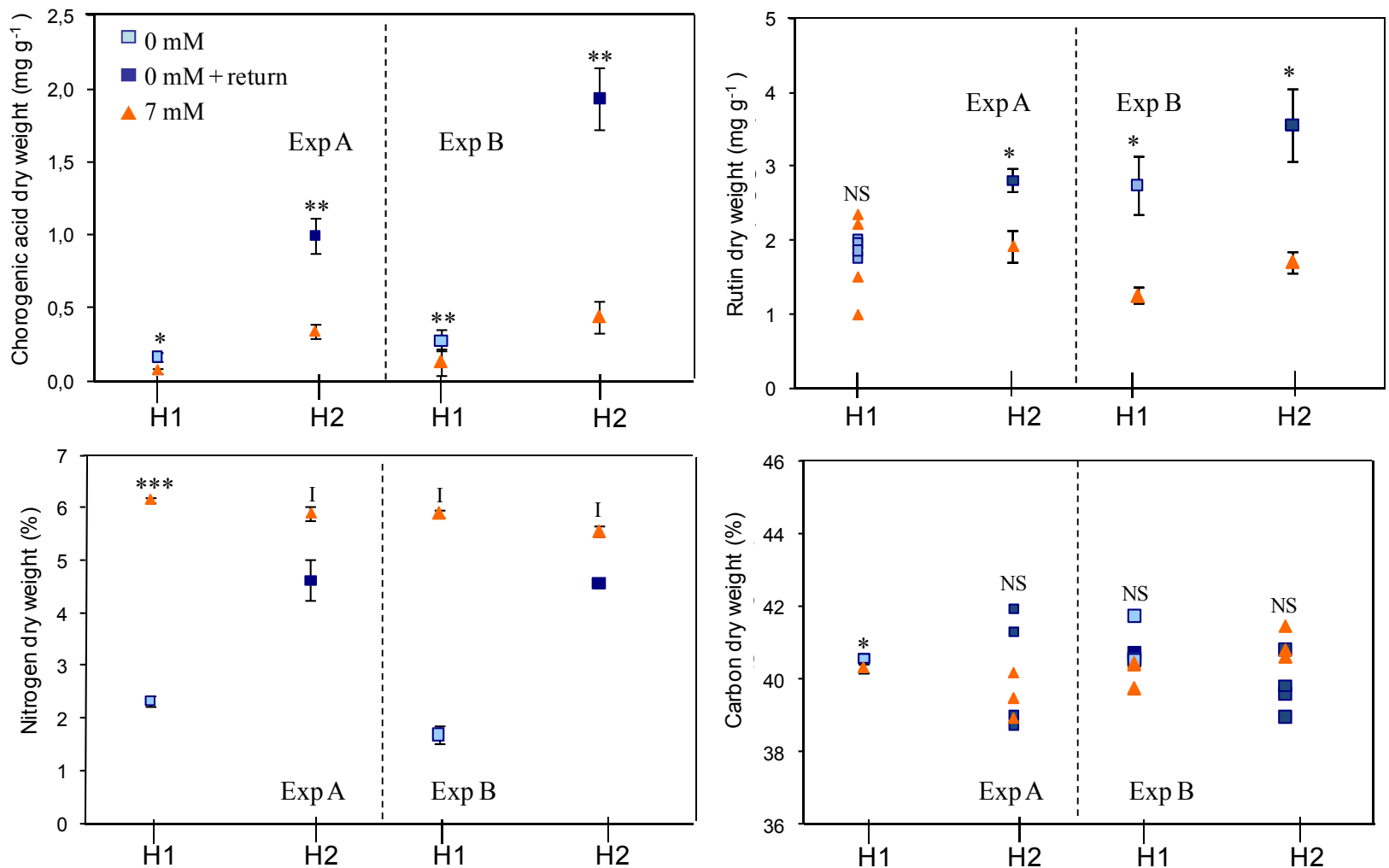

After the period of deprivation, chlorogenic acid levels were more than doubled in treated plants when compared with control plants (increase of $136 \%$ and $117 \%$ during experiments $\mathrm{A}$ and $\mathrm{B}$, respectively; Figure 4). Rutin levels in treated plants did not differ from those in control plants during experiment A (increase of 7\%), but were doubled in treated plants versus control plants in experiment $\mathrm{B}$ (increase of 119\%). These differences were further amplified after a return to a rich medium. Indeed, the chlorogenic acid contents found during both experiments continued to rise after a return to a rich medium (increase of $194 \%$ and $340 \%$ in experiments $\mathrm{A}$ and $\mathrm{B}$, respectively). This trend was also observed for rutin, levels of which increased by $50 \%$ and $110 \%$ respectively in experiments A and $\mathrm{B}$.

\section{Discussion}

\subsection{Effect of Nitrogen Deprivation on Plant Development}

Within a ten day period of nitrogen deprivation, the plants had developed a strategy for fresh matter distribution, with preferential growth of its root system biomass. This development strategy is frequently observed $[21,22]$ because a plant will make every effort to explore its cultivation medium to find the elements it is missing. Generally speaking, the production of fresh matter was almost reduced by half in treated plants, indicating that the deprivation duration was long enough to induce 
morphological modifications. The return to a rich medium for five days induced a resumption of growth but it was not enough to make up for the delay suffered during this period.

\subsection{Effect of Nitrogen Deprivation on Leaf Composition}

Although the tomato plants contained quite high levels of nitrogen (accumulated during the 10-day acclimatization period on a $7 \mathrm{mM} \mathrm{NO}_{3}{ }^{-}$medium), after just a few days of deprivation both their nitrogen status and metabolism were affected. Indeed we observed a very marked reduction in the percentage of nitrogen in tissues after the period of deprivation $(6 \mathrm{~g} \mathrm{~N}$ per $100 \mathrm{~g}$ dry matter in plants remaining at $7 \mathrm{mM}$ and approximately $2 \mathrm{~g} \mathrm{~N}$ per $100 \mathrm{~g}$ dry matter in treated plants), and an increase in the content of secondary metabolites (Figure 4). These observations confirmed the findings of previous studies using polyphenol concentrations as an indicator of plant nitrogen status [23].

During the period of deprivation, a modification to the nitrogen supply from the nutrient solution was accompanied by changes to secondary metabolism in the leaves. Levels of chlorogenic acid were doubled (increase of $136 \%$ and 117\%) in treated plants. Similar variations had previously been observed in Arabidopsis plantlets subjected to two days of deprivation [20], tomato plants subjected to 4 or 8 days of deprivation [14] and camomile plants subjected to 12 days of deprivation [24].

Scheible and co-workers [20] observed a marked rise in rutin concentrations, but in general the variations were smaller regarding the content in compounds synthesized at the start of, or midway through, the polyphenol biosynthetic pathway, such as hydroxycinnamic derivatives. During the present study, we also observed an increase in rutin levels during experiment $\mathrm{B}$, but it was the concentrations of chlorogenic acid, a hydroxycinnamic derivative occurring midway through the biosynthetic pathway, that were particularly affected. This difference in the type of compounds that accumulated may have been linked to the plants under study. Indeed, in Arabidopsis, hydroxycinnamic derivatives such as chlorogenic acid do not accumulate in the tissues, unlike what happens in the tomato [2,14].

The aim of this study was to determine if, after a return to more favourable cultivation conditions (7 $\mathrm{mM} \mathrm{NO}_{3}{ }^{-}$), a plant retained any metabolic stigma of a period of deprivation. During a previous study, a supply of $\mathrm{NO}_{3}{ }^{-}$to nitrogen-deprived Arabidopsis plantlets (at cotyledon stage) had caused a repression of genes involved in polyphenol synthesis that was detectable after less than 30 minutes and marked after 3 hours [20]. In addition, compounds whose synthesis had been stimulated by deprivation (cafeic acid, ferulic acid and rutin) remained at higher levels between 3 and 24 hours after the addition of nitrogen when compared with control, non-stressed plants. The results presented here confirm that even over a longer period of a return to a rich medium (five days) and in more developed plants ( 2 leaves tomato plants at the beginning of the experiment), the effects of stress remained visible: the levels of chlorogenic acid and rutin were higher in plants that had previously been deprived when compared with control plants.

Moreover, the content in rutin and chlorogenic acid in the treated plants strongly increased after a return to the rich medium compared to the control. To the best of our knowledge nothing has been established on how these compounds are catabolised or metabolised after their synthesis. So it may be possible that the phenolics previously synthesized remain in the plant for such a period. In addition, the recovery of nitrogen (as shown by increased leaf nitrogen content) may have permitted a strong enhancement of phenolics biosynthesis. Indeed, C-based secondary metabolites do not contain N, but 
the metabolic machinery necessary for their synthesis does need and does contain $\mathrm{N}$ [12]. Using the Growth Differentiation Balance Hypothesis [25], we may hypothesise that after the return to the rich medium, treated plants are still subjected to growth restriction but no more to photosynthesis limitation (see Figure 1 in [12]), so that more carbon is available for the synthesis of C-based compound such as phenolics.

Results presented here demonstrated that the accumulation of secondary metabolites persisted after a temporary nitrogen depletion, and consequently growing plants under non optimal conditions using temporary environmental restriction offer optimistic prospects for increasing plant resistance. Moreover it is possible to draw some parallels between data presented herein and what is already known regarding the response of secondary metabolism to biotic stress [4,26,27]. The defense mechanisms of plants against pathogens trigger a succession of phenomena. After recognizing the pathogen, a local response develops (hypersensitive response), followed by a global response at the scale of the whole plant (acquired systemic response) which endows it with long-term protection against future attacks. It is thus possible to imagine a similar behavior in plants when faced with an abiotic stress such as a deficit in mineral elements. For example, mechanisms such as enzyme synthesis, developing during an initial episode of stress, may induce a more intense response, i.e., an elevation of polyphenol levels that becomes more marked during a subsequent episode of deprivation. It would therefore be interesting to quantify polyphenol levels in a plant following several episodes of nitrogen deprivation.

\section{Experimental Section}

\subsection{Plant Materials, Growth Conditions and Nitrogen Treatments}

Tomato plants (Solanum lycopersicum cv Microtom) were grown under hydroponic conditions in a phytotron. The temperature in the growth chamber was $23-25{ }^{\circ} \mathrm{C}$ during the day and $18-20{ }^{\circ} \mathrm{C}$ at night, with relative humidity of $75 \%$ during the day and $70 \%$ at night. The mean photosynthetically active radiation (PAR) was $250 \mu \mathrm{mol} \mathrm{m} \mathrm{m}^{-2}$ and the photoperiod was 14 hours. Three weeks after germination in potting soil irrigated with water, plants with two pairs of leaves and $2 \mathrm{~cm}$ of roots were transferred to a container-based hydroponic cultivation system (1 plant/liter). The nutrient solution was continually aerated by a bubbling system and was renewed every three days (Tables 1 and 2). Two experiments, $\mathrm{A}$ and $\mathrm{B}$, were carried out.

Table 1. Composition of nutrient solutions as a function of nitrate contents of the medium. Gram weights of compounds required to prepare $1 \mathrm{~L}$ of solution.

\begin{tabular}{|c|c|c|c|c|c|c|c|c|}
\hline & $\begin{array}{l}\text { Compound/ } \\
\text { Weight (g) }\end{array}$ & $\mathrm{KH}_{2} \mathrm{PO}_{4}$ & $\mathrm{~K}_{2} \mathrm{SO}_{4}$ & $\begin{array}{c}\mathrm{Ca}\left(\mathrm{NO}_{3}\right)_{2} \\
4 \mathrm{H}_{2} \mathrm{O}\end{array}$ & $\begin{array}{c}\mathrm{CaSO}_{4}, \\
2 \mathrm{H}_{2} \mathrm{O}\end{array}$ & $\begin{array}{c}\mathbf{M g S O}_{4}, \\
7 \mathbf{H}_{2} \mathrm{O}\end{array}$ & $\begin{array}{c}\text { EDTA- } \\
\mathrm{Fe}, \\
\mathbf{1 H}_{2} \mathrm{O}\end{array}$ & $\begin{array}{l}\text { Oligoelements } \\
(\mathrm{mL} / \mathrm{L})\end{array}$ \\
\hline \multirow{2}{*}{$\mathrm{NO}_{3}^{-}(\mathrm{mM})$} & $\mathbf{0}$ & \multirow{2}{*}{0.136} & \multirow{2}{*}{0.174} & 0 & 0.603 & \multirow{2}{*}{0.37} & \multirow{2}{*}{0.017} & \multirow{2}{*}{0.1} \\
\hline & 7 & & & 0.827 & 0 & & & \\
\hline
\end{tabular}


Table 2. Composition of the oligoelement solution. Gram weights of compounds required to prepare $1 \mathrm{~L}$ of solution.

\begin{tabular}{lccccccc}
\hline Compound & $\mathbf{M o O}_{3}$ & $\begin{array}{c}\mathbf{M n C l}_{2}, \\
\mathbf{4 H}_{\mathbf{2}} \mathbf{O}\end{array}$ & $\begin{array}{c}\mathbf{Z n S O}_{4}, \\
\mathbf{7 H}_{\mathbf{2}} \mathbf{O}\end{array}$ & $\begin{array}{c}\mathbf{C u S O}_{4}, \\
\mathbf{5 H}_{\mathbf{2}} \mathbf{O}\end{array}$ & $\mathbf{H}_{\mathbf{3}} \mathbf{B O}_{\mathbf{3}}$ & $\mathbf{F e S O}_{4}$ & $\mathbf{N a}_{\mathbf{2}} \mathrm{EDTA}$ \\
\hline Weight $(\mathrm{g})$ & 0.40 & 23.06 & 9.32 & 1.18 & 12.75 & 29.87 & 59.56 \\
\hline
\end{tabular}

Firstly, sixteen plants were placed on the rich medium containing $7 \mathrm{mM} \mathrm{NO}_{3}^{-}$for 10 days (Figure 2). The plants were then separated into two groups of eight plants and transferred to two media: a rich medium containing $7 \mathrm{mM} \mathrm{NO}_{3}{ }^{-}$(referred to hereinafter as $7 \mathrm{mM}$ ) and a deficient medium containing no $\mathrm{NO}_{3}{ }^{-}$(referred to as $0 \mathrm{mM}$ ). The plants remained on these two media for 10 days during the first experiment (Experiment A) and 19 days during the second experiment (Experiment B). At the end of this period, four plants that had been subjected to deprivation (referred as treated plant) and four control plants were harvested. The other eight plants remained under hydroponic conditions for a further five days, during which they were all grown again on a nitrogen-rich solution $(7 \mathrm{mM})$ until they were harvested (in the text this period is referred to as the period of return to normal). Lovdal and coworkers [14] observed a more marked increase in flavonoids after 8 days compared to 4 days of nitrogen depletion. In order to maximize plant responses to nitrogen depletion, our plants were submitted to 10 days depletion (Experiment A) and then we doubled this time in experiment B (19 days of depletion).

\subsection{Plant Harvesting, Sampling and Chemical Analyses}

At each harvest, four plants per treatment (two containers) were collected. All fresh matter was determined by organ type: leaves, stems and roots. For chemical analyses, only the leaves were retained, frozen into liquid nitrogen and stored at $-20{ }^{\circ} \mathrm{C}$ until freeze-drying. Following this procedure, the samples were weighed to determine the dry matter (DM) and reduced to powder using a ball mill (MM200, Restch, France).

The percentages of total carbon and nitrogen were obtained from $5 \mathrm{mg}$ DM using gas chromatography with a carbon/nitrogen auto-analyzer (Flash EA 1112, Thermo Finnigan, USA). Polyphenols were assayed by HPLC on $50 \mathrm{mg}$ DM, according to the method described in [28]. The samples were analyzed using a Beckman System Gold instrument consisting of a 508 autosampler and a dual wavelength detector (module 166), set at $330 \mathrm{~nm}$ for quantifications. Chromatographic separations were performed on a Lichrospher RP-18 end-capped column $(4 \times 250 \mathrm{~mm}, 5 \mu \mathrm{m}$, Merck, Darmstadt, Germany) fitted with a Lichrospher RP-18 guard column ( $5 \mu \mathrm{m}$, Merck). The mobile phase was a binary solvent system consisting of (A) water adjusted to $\mathrm{pH} 2.6$ with orthophosphoric acid and (B) methanol. The gradient (from 3\% to $60 \%$ of B in $180 \mathrm{~min}$ ) was eluted at a flow rate of $0.5 \mathrm{~mL} \mathrm{~min}^{-1}$ at room temperature. Compounds were identified by their absorption spectra (Figure 5). All compounds were correctly separated, and quantification was based on peak surface area, referred to as a standard curve. Phenolic contents were expressed as mg per g Dry Weight (DW). Taxifolin (50 $\mu \mathrm{L}$ of a solution at $2 \mathrm{mg} \cdot \mathrm{mL}^{-1}$ methanol, Extrasynthèse, Lyon, France) was added as an internal standard. Rutin and chlorogenic acid standards were purchased from Sigma (Sigma, Saint Quentin-Fallavier, France). 
Figure 5. Chromatogram and UV spectra of phenolic compounds found in tomato leaves (cv Microtom). Detection was at $330 \mathrm{~nm}$, Taxifolin is added as an internal standard.

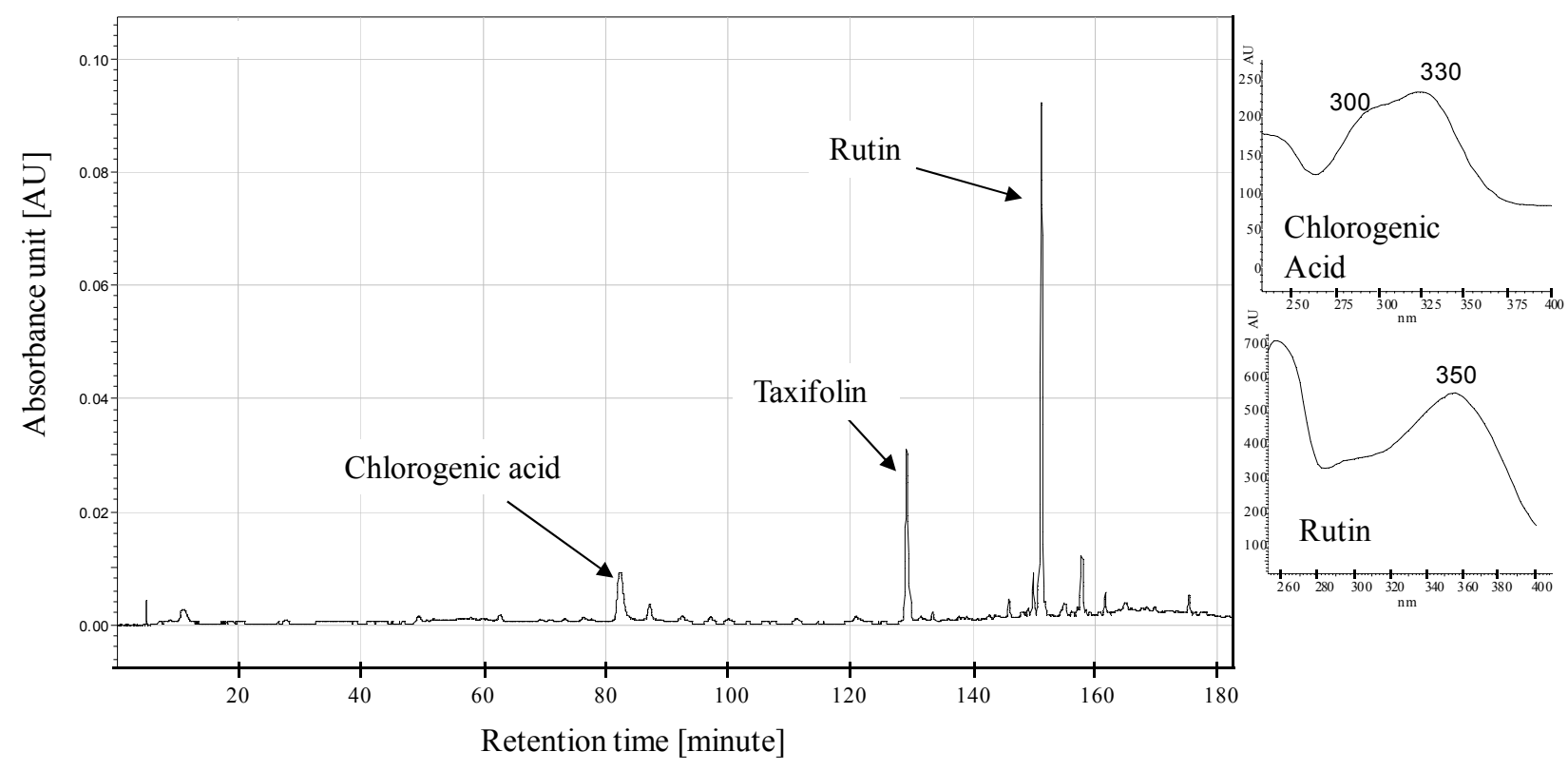

At each harvest and for each experiment we performed a two-way analysis of variance (ANOVA) using XLSTAT (Addinsoft, France) to compare control and deprived plants. The effect of nitrogen supply, the container, as a block effect, and the interaction between them were considered. The data shown on the figure are means calculated on the four plants per treatment, \pm standard error (SE). When the growth conditions had a significant effect i.e., container effect observed in the ANOVA analyses, the four plants per treatment were shown.

The differences obtained between the treatments were expressed as percentage variations calculated versus control plants grown on a nitrogen-rich medium throughout the study period.

\section{Conclusions}

The present work reveals part of the tomato plant strategy when submitted to a temporary nitrogen deprivation. A nitrogen deprivation period of 10 or 19 days induced a persistent effect on the phenolics content in leaves of tomato plants. Indeed we quantified a higher content of phenolics in leaves of tomato plants grown with no nitrogen in comparison to control plants grown with $7 \mathrm{mM} \mathrm{NO}_{3}^{-}$. Moreover, the content of chlorogenic acid and rutin remained higher in plants that had previously been deprived compared to the control when all these plants were further cultivated on a nitrogen-rich medium for 5 days.

Furthermore, during these experiments, the duration of nitrogen stress (10 to 19 days) engendered a marked reduction in plant biomass production. It would therefore be interesting to test the impact of occasional nitrogen deprivation on plants that have already developed larger leaf area in order to determine whether such nitrogen stresses are sufficient to enhance the defenses of vegetative plant parts without affecting fruit yield. 


\section{References}

1. Wilkens, R.T.; Spoerke, J.M.; Stamp, N.E. Differential responses of growth and two soluble phenolics of tomato to resource availability. Ecology 1996, 77, 247-258.

2. Mittelstra $\beta$, K.; Treutter, D.; Pleß1, M.; Heller, W.; Elstner, E.F.; Heiser, L. Modification of primary and secondary metabolism of potato plants by nitrogen application differentially affects resistance to Phytophtora infestans and Alternaria solani. Plant Biol. 2006, 8, 653-661.

3. Niggeweg, R.; Michael, A.; Martin, C. Engineering plants with increased levels of the antioxidant chlorogenic acid. Nat. Biotechnol. 2004, 22, 746-754.

4. Treutter, D. Significance of flavonoids in plant resistance: A review. Environ. Chem. Lett. 2006, 4, 147-157.

5. Hoffland, E.; Dicke, M.; Van Tintelen, W.; Dijkman, H.; Van Beusichem, M.L. Nitrogen availability and defense of tomato against two-spotted spider mite. J. Chem. Ecol. 2000, 26, 2697-2711.

6. Johnson, K.S. Plant phenolics as radical scavengers in the context of insect (Manduca sexta) hemolymph and midgut fluid. J. Agric. Food Chem. 2005, 53, 10120-10126.

7. Johnson, K.S.; Felton, G.W. Plant phenolics as dietary antioxidants for herbivorous insects: A test with genetically modified tobacco. J. Chem. Ecol. 2001, 27, 2579-2597.

8. Fujita, M.; Fujita, Y.; Noutoshi, Y.; Takahashi, F.; Narusaka, Y.; Yamaguchi-Shinozaki, K.; Shinozaki, K. Crosstalk between abiotic and biotic stress responses: A current view from the points of convergence in the stress signaling networks. Curr. Opin. Plant Biol. 2006, 9, 436-442.

9. Lillo, C.; Lea, U.S.; Ruoff, P. Nutrient depletion as a key factor for manipulating gene expression and product formation in different branches of the flavonoid pathway. Plant Cell Environ. 2008, 31, 587-601.

10. Urbanczyk-Wochniak, E.; Fernie, A.R. Metabolic profiling reveals altered nitrogen nutrient regimes have diverse effects on the metabolism of hydroponically-grown tomato (Solanum lycopersicum) plants. J. Exp. Bot. 2005, 56, 309-321.

11. Dumas, Y.; Dadomo, M.; Di Lucca, G.; Grolier, P. Review of the influence of major environmental and agronomic factors on the lycopene content of tomato fruit. Acta Hortic. 2002, 579, 595-601.

12. Le Bot, J.; Benard, C.; Robin, C.; Bourgaud, F.; Adamowicz, S. The "trade-off" between synthesis of primary and secondary compounds in young tomato leaves is altered by nitrate nutrition: experimental evidence and model consistency. J. Exp. Bot. 2009, 60, 4301-4314.

13. Stout, M.J.; Brovont, R.A.; Duffey, S.S. Effect of nitrogen availability on expression of constitutive and inducible chemical defenses in tomato, Lycopersicon esculentum. J. Chem. Ecol. 1998, 24, 945-963.

14. Lovdal, T.; Olsen, K.M.; Slimestad, R.; Verheul, M.; Lillo, C. Synergetic effects of nitrogen depletion, temperature, and light on the content of phenolic compounds and gene expression in leaves of tomato. Phytochemistry 2010, 71, 605-613.

15. Le Bot, J.; Jeannequin, B.; Fabre, R. Impact of N-deprivation on the yield and nitrogen budget of rockwool grown tomatoes. Agronomie 2001, 21, 341-350.

16. Toth, V.R.; Meszaros, I.; Palmer, S.J.; Veres, S.; Precsenyi, I. Nitrogen deprivation induces changes in the leaf elongation zone of maize seedlings. Biol. Plant. 2002, 45, 241-247. 
17. Warner, J.; Zhang, T.; Hao, X. Effects of nitrogen fertilization on fruit yields and quality of processing tomatoes. Can. J. Plant Sci. 2004, 84, 865-871.

18. Murshed, R.; Lopez-Lauri, F.; Keller, C.; Monnet, F.; Sallanon, H. Acclimation to drought stress enhances oxidative stress tolerance in Solanum Lycopersicum L. fruits. Plant Stress 2008, 2,145-151.

19. Walter, J.; Nagy, L.; Heinb, R.; Rascherc, U.; Beierkuhnleinb, C.; Willnerd, E.; Jentsche, A. Do plants remember drought? Hints towards a drought-memory in grasses. Environ. Exp. Bot. 2011, 71, 34-40.

20. Scheible, W.R.; Morcuende, R.; Czechowski, T.; Fritz, C.; Osuna, D.; Palacios-Rojas, N.; Schindelasch, D.; Thimm, O.; Udvardi, M.K.; Stitt, M. Genome-wide reprogramming of primary and secondary metabolism, protein synthesis, cellular growth processes, and the regulatory infrastructure of Arabidopsis in response to nitrogen. Plant Physiol. 2004, 136, 2483-2499.

21. Brouwer, R. Distribution of dry matter in the plant. Neth. J. Agric. Sci. 1962, 10, 361-376.

22. Lemaire, F. Comparative action of nitrogen supply on growth of root system and aerial parts of plants. Ann. Agron. 1975, 26, 59-73.

23. Cartelat, A.; Cerovic, Z.G.; Goulas, Y.; Meyer, S.; Lelarge, C.; Prioul, J.L.; Barbottin, A.; Jeuffroy, M.H.; Gate, P.; Agati, G.; et al. Optically assessed contents of leaf polyphenolics and chlorophyll as indicators of nitrogen deficiency in wheat (Triticum aestivum L.). Field Crops Res. 2005, 91, 35-49.

24. Kovacik, J.; Klejdus, B.; Backor, M. and Repcak, M. Phenylalanine ammonia-lyase activity and phenolic compounds accumulation in nitrogen-deficient Matricaria chamomilla leaf rosettes. Plant Sci. 2007, 172, 393-399.

25. Herms, D.A.; Mattson, W.J. The dilemma of plants: to grow or defend. Quart. Rev. Biol. 1992, 67, 283-335.

26. Baidez, A.G.; Gomez, P.; Del Rio, J.A.; Ortuno, A. Antifungal capacity of major phenolic compounds of Olea europaea L. against Phytophthora megasperma Drechsler and Cylindrocarpon destructans (Zinssm.) Scholten. Physiol. Mol. Plant Pathol. 2006, 69, 224-229.

27. Lattanzio, V.; Lattanzio, V.M.T.; Cardinali, A. Role of Phenolics in the Resistance Mechanisms of Plants against Fungal Pathogens and Insects. In Phytochemistry: Advances in Research; Research Signpost: Kerala, India, 2006; pp. 23-67.

28. Benard, C.; Gautier, H.; Bourgaud, F.; Grasselly, D.; Navez, B.; Caris-Veyrat, C.; Weiss, M.; Genard, M. Effects of low nitrogen supply on tomato (Solanum lycopersicum) fruit yield and quality with special emphasis on sugars, acids, ascorbate, carotenoids, and phenolic compounds. J. Agric. Food Chem. 2009, 57, 4112-4123.

(C) 2011 by the authors; licensee MDPI, Basel, Switzerland. This article is an open access article distributed under the terms and conditions of the Creative Commons Attribution license (http://creativecommons.org/licenses/by/3.0/). 